\title{
Editorial
}

\section{Reprogramming: So simple, so complex}

\author{
A Zeuner ${ }^{*, 1}$ and R De Maria ${ }^{2}$ \\ Cell Death and Differentiation (2012) 19, 1253-1254. doi:10.1038/cdd.2012.64
}

The seminal discovery by Takahashi and Yamanaka ${ }^{1}$ that specialized somatic cells can be reverted into pluripotent embryonic progenitors through the process of reprogramming has revolutionized the concept of cell identity and paved the way for the development of new cellular therapies. The inherent pluripotency of reprogrammed cells (iPS, induced pluripotent stem cells) offers potential applications for a plethora of cell-autonomous disorders, as demonstrated by several studies that generated iPS-based disease models for neurological, hematological, cardiovascular, metabolic and endocrine conditions (reviewed in Grskovic et al. ${ }^{2}$ ). An increasing number of reports confirm the promising clinical applications of reprogramming, such as the recent demonstration that fibroblasts can be directly converted into neural stem cells with a defined set of transcription factors, theoretically providing an unlimited source of cells for nervous system regeneration. ${ }^{3}$ In the meanwhile, experimental researchers restlessly work to dissect the reprogramming process, in the attempt to disclose the mechanisms responsible for cell plasticity.

The article by Nemajerova et al. in this issue of Cell Death and Differentiation provides new insights into the mechanisms of reprogramming, showing that selected subpopulations of mouse embryonic fibroblasts (MEFs) are more prone to reprogramming and that a two-factor combination (Oct4/ Sox2 or Oct4/KIf4) is sufficient to induce a transient de-differentiation state from which iPS emerge in a stochastic fashion (Figure 1).

MEFs traditionally represent the starting cell population for mechanistic studies on reprogramming. Fibroblasts have been previously described as a dynamic cell population. ${ }^{4}$ However, their heterogeneity has never been exploited as a resource for iPS generation. Nemajerova et al. showed that reducing MEF heterogeneity by means of cell sorting for specific surface markers (Thy1 and Sca1) yields discrete subpopulations that can be reprogrammed with a minimal combination of factors. Specifically, the Oct4/Sox2 (OS) combination was more effective than the Oct4/KI4 (OK) combination in inducing the expression of pluripotencyassociated gene modules, according to the fact that Oct4 and Sox2 can bind DNA as a heterodimer.

Separating MEF fractions with different reprogramming competency likely reflects the existence of cells with different degrees of differentiation within the fibroblast population.

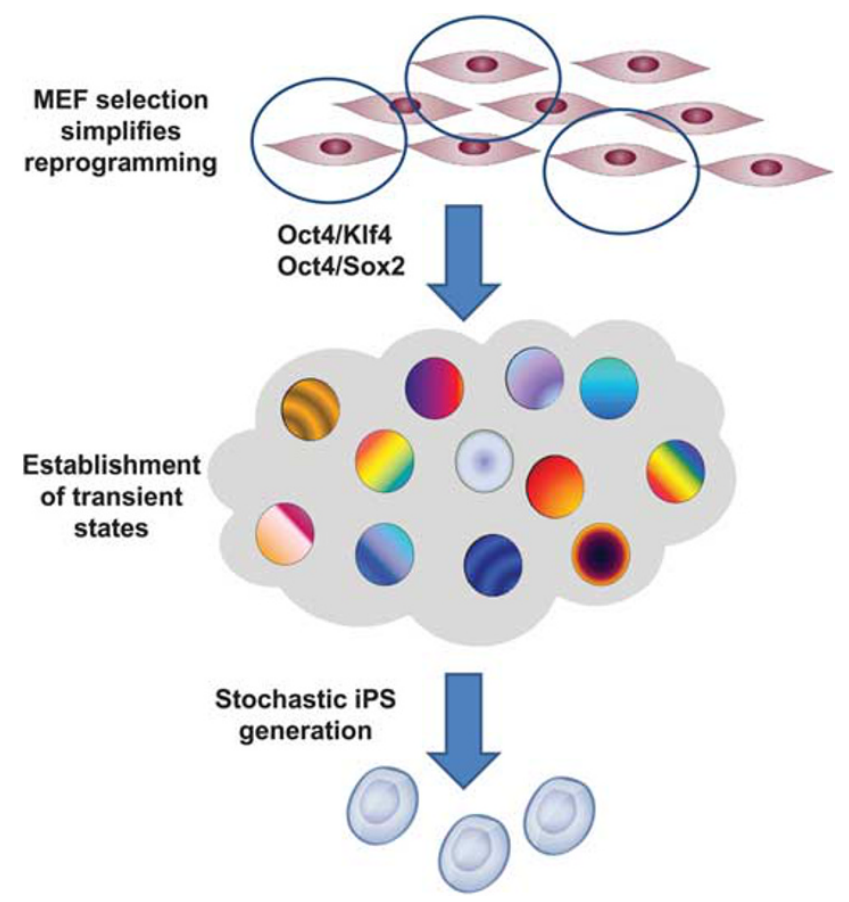

Figure 1 Selected MEF subpopulations can be reprogrammed with a two-factor combination (Oct4/Sox2 or Oct4/Klf4), inducing a transient dedifferentiation state from which iPS emerge in a stochastic fashion

In fact, it was previously demonstrated that less differentiated cells (e.g. neural stem cells) can be reprogrammed with fewer factors. ${ }^{5}$ Because reprogramming is increasingly viewed as a stochastic process, it is also plausible that undifferentiated cell populations are more amenable to reprogramming due to a higher level of instability in response to internal or external stimuli. 6,7

One of the most intriguing aspects of reprogramming, which is also covered by Nemajerova's work, is the process by which cells convert to a pluripotent state. While several studies showed that reprogramming occurs through a series of intermediate steps, ${ }^{8}$ the nature and succession of such steps remains a matter of debate. Some investigators suggested that conversion into a pluripotent state involves a fixed

'Department of Hematology, Oncology and Molecular Medicine, Istituto Superiore di Sanità, Rome, Italy and 'National Cancer Institute 'Regina Elena', Via Elio Chianesi 53, Rome, Italy

*Corresponding author: A Zeuner, Department of Hematology, Oncology and Molecular Medicine, Istituto Superiore di Sanita, Viale Regina Elena 299, Rome 00161, Italy. Tel: + 396 49906060; Fax: + 396 49387087; E-mail: a.zeuner@iss.it 
stepwise path entailing mesenchymal-epithelial transition, ${ }^{9}$ while others argue in favor of a stochastic process that involves a random drift of cell states. ${ }^{10}$ The findings by Nemajerova et al. support the latter view of reprogramming, showing that either the OS or OK factor combination disrupts cellular homeostasis, establishing transient differentiation states characterized by the expression of mixed lineage markers. Across this 'boiling pot' of different cell states, iPS are stochastically generated at a relatively low frequency.

The low efficiency of reprogramming is one of the major limitations that restrict a future clinical use of iPS. Countless efforts have been made to identify chemical or genetic factors that favor the reacquisition of pluripotency in somatic cells. Small-molecule compounds, microRNAs, histone demethylases and chromatin-modifying enzymes have all been shown to act as reprogramming modulators. ${ }^{11-13}$ In Nemajerova's study, reprogramming efficiency reaches $2 \%$ with selected MEF populations, which can be considered a reasonable efficiency considering that it has been obtained with a reduced number of factors. However, although it was demonstrated that all cells in a given population undergo reprogramming with sufficient time and the right conditions, ${ }^{10}$ iPS generation still remains a process difficult to control, possibly due to its stochastic nature.

Reduction in the number of reprogramming factors has been repeatedly suggested as an approach to minimize chromosomal disruption in iPS and the subsequent risk of malignant transformation. Several studies demonstrated that a reduced set of reprogramming factors is sufficient to generate iPS, albeit at lower efficiency. ${ }^{5,14-16}$ However, a recent study warned against 'minimalist' approaches to reprogramming, showing that iPS obtained with a reduced number of factors (down to a single factor, Oct4) not only are generated at lower efficiency but also show functional defects upon differentiation into neuronal cells. ${ }^{17}$ In this context, it would be important to determine whether iPS generated from selected MEF subpopulations with two-factor combinations maintain an intact ability to generate functional differentiated cells.

The realization that cellular identity is malleable has opened huge perspectives for cellular therapies and in vivo regeneration. In parallel, new discoveries also indicate the persistence of 'complexity zones' that strongly influence the outcome of somatic cell reprogramming. Every progress in exploring such zones should be seen as a step that brings iPS closer to the patients' bedside.

\section{Conflict of Interest}

The authors declare no conflict of interest.

1. Takahashi K, Yamanaka S. Cell 2006; 126: 663-676.

2. Grskovic M et al. Nat Rev Drug Discov 2011; 10: 915-929.

3. Han DW et al. Cell Stem Cell 2012; 10: 465-472.

4. Phan SH. Proc Am Thorac Soc 2008; 5: 334-337.

5. Kim JB et al. Nature 2009; 461: 649-653.

6. MacArthur BD, Please CP. Oreffo ROC. PLoS One 2008; 3: e3086.

7. Loh KM, Lim B. Cell Stem Cell 2011; 8: 363-369.

8. Stadtfeld M et al. Cell Stem Cell 2008; 2: 230-240.

9. Li R et al. Cell Stem Cell 2010; 7: 51-63.

10. Hanna J et al. Nature 2009; 462: 595-601.

11. Huangfu D et al. Nature Biotech 2008; 26: 795-797.

12. Wang T et al. Cell Stem Cell 2011; 9: 575-587.

13. Onder TT et al. Nature 2012; 483: 598-602.

14. Huangfu D et al. Nat Biotech 2008; 26: 1269-1275.

15. Kim JB et al. Cell 2009; 136: 411-419.

16. Zhu S et al. Cell Stem Cell 2010; 7: 651-655.

17. Löhle M et al. Stem Cells 2012; 30: 570-579. 